\title{
Research on the Spread and Inheritance of Huayin Old Cavity Culture and Art
}

\author{
Shan $\mathrm{HaO}^{1, \mathrm{a}}$ \\ ${ }^{1}$ Xi'an University, Xi'an, Shaanxi, China, 710065 \\ aemail,
}

Keywords: Spread, Inheritance, Huayin Laojian, Culture and Art

\begin{abstract}
Huayin Old Cavity is the first batch of national intangible cultural heritage in Chinese, it is widely absorbed in Shaanxi Guanzhong folk music elements to Qin sound as the basis of phonology, and it is Chinese northern shadow art rare "living fossil." This paper mainly elaborates the artistic characteristics of the old cavity, and on the basis of analyzing the present situation of Huayin old cavity transmission and inheritance, puts forward some superficial opinions and suggestions on the reform and development of Huayin old cavity.
\end{abstract}

\section{Introduction}

Huayin old cavity is popular in Huayin City, Shaanxi Province, is quite a local characteristics of a shadow opera, also known as Cloth light shadow, the old cavity light shadow. Huayin old cavity has a long history, to the tragic ancient tunes, vigorous singing, rough and unrestrained style, high-pitched wide range of characteristics, exudes the unique charm of the northwest loess. With the diversification of social and cultural development, more and more emerging fashion culture and entertainment in the form of people's lives, Huayin old cavity where the opera gradually embarked on the decline of the way. In order to inherit and develop, Huayin old cavity for a bold change, the stage from behind the scenes moved to the front, to participate in drama, film and other performances, and pop singer cooperation, so that the old cavity and the old cavity artists gradually from the village to the international City, more and more for the audience favorite. But the old cavity of the heritage and development of the existence of various bottlenecks, need to be resolved.

\section{The Origin of Huayin Old Cavity}

The old cavity is named after Huayin, Huayin is located in the Qin Yu Jin provinces transfer the land, according to the Qinling Mountains, north near the Wei Luo, only a hundred kilometers from Xi'an, the "three Qin Road, eight provinces and rivers" reputation The Huayin Spring and Autumn set up town, war country home county, the last century to withdraw the county set up the city, has two thousand years of history. Huayin terrain more flat, fertile soil, suitable for crop growth, agricultural economy developed for the birth of the old cavity laid the economic foundation. Dynasties emperors will phase, merchants of the Huashan worship activities for Huayin brought a variety of cultural forms, for the birth of the old cavity to provide a cultural soil.

The old cavity originated in Shuangquan Village, Huayin City, but there are two different views on the origin of the time. First, the old cavity originated in the Western Han Dynasty. During the Western Han Dynasty, Shuangquan village near the capital granary and responsible for grain transport administration, during the river water transport is unusually busy, the labor churches come and go, slender pull towels, a leader and, and with a piece of wood rhythmically hit the ship board Enhance the appeal, so that the formation of the old cavity of the prototype. Later, Fu Fu rest, and garrison soldiers singing with the siren, it was suggested that with rags cut adult style with singing, experienced a long time baptism, the formation of the shadow of the old cavity. Second, the old cavity originated in the Ming and Qing Dynasties. Some old cavity artists believe that the old chamber by the storytellers evolved, before the performance of the book before the use of wood knocked on the table, and the old cavity used as a gavel, played a warning role in the Ming and Qing Dynasties Shuangquan village performance evaluation book Artists, and later commentary 
artists feel that the storyteller cannot attract people, so to improve, to the storyteller plus musical instruments, while playing side to say, slowly formed the old cavity.

\section{The Artistic Characteristics of Huayin Old Cavity}

The original ecology is the biggest artistic feature of Huayin old cavity. "In Shaanxi, the most able to reflect the Qin character of the shadow of singing, it is the number of Huayin old cavity." Very often, artists and their singing, as it is the lyrics "roar" out. Because of its strong original ecological singing, Huayin old cavity is affectionately known as "Oriental rock" also by Chinese and foreign audience. In Shaanxi Guanzhong popular opera, Huayin old cavity in the selection, melody, singing, clothing, props, etc., are more close to the masses of production and life. For example, the old chamber performances, the actors are not deliberately make-up, there is no complex performance costumes. In the performance of the action, the old cavity art people also showed great randomness, and some sitting on the bench, stool, and some leaning on the pillars, walls, and even squatting on the stage or sitting on the floor. Female artists on the stage are often hand-held spindle imitation spinning weaving, or hand soles imitate the needle, this kind of daily life with a high degree of uniform form of performance in our local opera is very rare. Life atmosphere strong original ecological style, it is precisely the Huayin old cavity where the unique charm.

The origin of music is another significant feature of Huayin old cavity. Huayin old cavity of the main accompaniment instruments are cowhide chord piano, silk chord board, large, horse, jujube board, gongs, gongs, gongs, gongs, drums, drums, drums, gongs, , Bell and so on. It is worth noting that the old cavity art people never use suona for accompaniment, more attention and preference for percussion instruments. In the music structure, the old chamber accompaniment music preferences using overlapping phrases, dramatic effects. Artists beat the jujube board imitate the horses screaming sound horse, the audience as if exposure to the iron horse Jin Ge, people shouting the hiss of the battlefield, Dunsheng bleak, excited feeling.

Huayin old cavity singing has a strong language, voice and lyrics closely, cavity by the word students, sound and color, and the cavity is full, and said, read and sing intertwined in the same paragraph, showing the sound art the early development of the sound from the opera to the obvious traces of the transition. Huayin old cavity belongs to the cavity of the cavity structure with a strong northern cavity drama music characteristic. Plate cavity, also known as plate change body, refers to a basic tune based on the speed, beat, intensity, melody and other elements of change in the melody and the body to take the expansion and contraction of the way, and gradually evolved a Series of different plate, forming a set of a variety of drama performance with the singing, this singing is generally known as the board cavity. Huayin old cavity of the basic plate with a pull wave, Adagio, water, crying board, rolling plate, flying board, flower station, walking field, etc., with strong customs in the local characteristics.

The singleness of the material is a major feature of the old cavity. Due to the closeness of the family heritage, Huayin old cavity is more concerned about the historical theme, very few performances of the new era of new tracks. Huayin old age is good at the performance of the war scenes, longer than the narrative of the ancient historical figures of the hero story, from the "Eastern Zhou Dynasty", "Gods", "Romance of the Three Kingdoms" "said Tang Jiyi" and other traditional folk books, book search for inspiration. Again, "by Zhao Yun" "take Xichuan" "out of five" and other famous tracks, all reflect the Huayin old cavity of this feature.

\section{The Heritage Development Bottleneck of Huayin Old Cavity}

In recent years, Huayin old chamber by the audience at home and abroad like, under the protection of the national intangible heritage, made gratifying achievements and development, but in the multi-cultural impact, the old cavity facing the problem is not fundamentally To solve the development of its heritage into a bottleneck.

Huayin old cavity after the lack of people, faced with the risk of taking the skills. Huayin old age when the peak, Shuangquan village hundreds of people, there are more than ten troupe, now, the old 
chamber singer only a few people only. Among them, the oldest more than 70 years old, the youngest also over half a year, coupled with Huayin old chamber followed the family style of learning, inheritance is limited to Zhang's family, not pass the surname, the current lack of predicament, urgent to be a talent reserve. In order to solve this problem, Huayin government in 2008 organized Huayin old cavity training courses to attract more people into the old cavity performance team. A month's time only recruited more than 40 people, and mostly over half a hundred years, there is no basis, and really insisted down only a few people. Old cavity artist Zhang Ximin once said: "In order to let their grandchildren learn the old cavity, every time they are coaxed." Visible, Huayin old cavity in the lack of basic groups in the young group, it is difficult to attract young people to learn Huayin old Cavity, the successor of the situation is getting worse.

Huayin old cavity literature loss script save situation worrying. Huayin old cavity traditional repertoire colorful, from the Qing Dynasty to the new China was established, Zhang clan has been kept with the script, but because of age, coupled with personal preservation, so save the script is not ideal, the paper yellow There are stains, foot damage is very serious, inside the writing is also blurred. In addition, many traditional repertoire singing by the old artists word of mouth, and no script handed down, resulting in the current Huayin old cavity literature loss is serious, the script save situation worrying.

Huayin old chamber concept stale, it is difficult to meet the needs of the audience. Huayin old cavity many traditional repertoire due to the impact of the times produced, its advocacy respected world view, outlook on life, values are relatively backward. Under the impact of modern multiculturalism, it is difficult to meet the growing spiritual and cultural needs of the audience, so that Huayin old cavity is now in the endangered state, survival and development of a great crisis.

Huayin old cavity audience face narrow, the market operation to be perfect. In recent years, Huayin old cavity in the market has made some achievements, repeated the country, and even the international arena, but faced with the risk of away from the general audience. At present, Huayin old chamber dealer prices rise, many ordinary viewers discouraged, it is difficult to personally experience the unique charm of Huayin old cavity. For example, Huashan scenic old performance tickets up to 180 yuan, far beyond the level of the general audience consumption. In addition, new media continue to emerge, so that the audience lost serious, especially young audience.

\section{The Development Countermeasures of Huayin Old Cavity}

Break the family heritage model, reserve follow-up talent. Family heritage model is Huayin old cavity follow-up talent shortage of the main obstacles, the old cavity if you want to reproduce the past brilliant, breaking the family closed heritage is a top priority. Huayin old cavity of the heritage should not be confined to the family of Zhang, should be under the guidance of the relevant government departments, and actively absorb the love of the old art of the opera lovers and have a certain basis for singing or singing talent, the establishment of the old cavity training class, the use of mentoring system of teaching mode, training successor. Huayin old cavity artist can also cooperate with the theater academy, theater institutions set up Huayin old cavity professional, hire the old cavity artists as a mentor, to guide students how to interpret the old class classic repertoire, at the same time, institutions will learn to improve the old cavity The overall quality of the subsequent power.

Put human and material resources, to protect the old cavity script. Huayin old cavity for the preservation of the status quo worry about the status quo, the relevant government departments should invest a certain amount of human, material and financial resources, organization of personnel to collect the old script, sorting. The establishment of Huayin old cavity art museum will collect and organize the precious old script and performance props and other professional institutions for custody, to avoid personal damage caused by improper damage or loss. At the same time, the relevant government departments should actively use the modern new media technology, the old chamber artist's traditional classic repertoire singing and shadow performances of audio and video recording, publishing audio and video products; will organize a good script, literature and other books published, so that it can protect the old cavity script, but also can spread the old cavity 
art.

Use modern means of communication improve the operation of the market. In recent years, new media has emerged, because of its communication convenience, diversity, efficiency and other characteristics of the traditional culture of heritage and development provides more opportunities. New media background, everyone can participate in mass communication, communicators can blog, micro-blogging, We-Chat and other forms, to share their own knowledge, experience and ideas. Therefore, Huayin old cavity should actively use the modern means of transmission, will be excellent repertoire performances, singers, shadow production, shadow play and other video or audio, the use of the advantages of new media network, and actively promote the old cavity art. At the same time, Huayin old cavity should set up a special propaganda operation team, the use of modern media improve the marketing mechanism, to seize the cultural market. In addition, Huayin old cavity should be with the famous tourist attractions in Shaanxi, Huayin old cavity in Shaanxi famous scenic performances normalized, the fare rationalization, performing specialization, at the same time, the old cavity artists should go into the primary school and college school, let the children, young people better understand the old cavity art, experience the old chamber charm.

\section{Conclusion}

As one of the oldest operas in China, Huayin old cavity has such a problem on the road of inheritance and development, but we have reason to believe that under the emphasis and fostering of relevant government agencies, we can break the traditional family inheritance model put a lot of manpower and material resources, and modern art forms of integration, to seek their own innovation, with modern media, Huayin old cavity will continue to spread in the domestic and international stage shine.

\section{Acknowledgements}

Fund Project: Shaanxi Provincial Social Science Fund Project. Study on the Spread and Inheritance of Huayin Old Cavity Culture and Art. Project ID: 2016J003

\section{References}

[1] Liu Xinyi. Historical inheritance and the present evolution - On Huayin old chamber singing style [J]. Singing Art. 2017 (02)

[2] Qian Suwei. Give more to the "Huayin old chamber" show face opportunities [J]. Communist Party members (Hebei) .2016 (11)

[3] Bai Ying. Huayin old lakes on the rock of the rock [J]. Today's nation. 2016 (04)

[4] Feng Jiao. On Huayin old cavity protection status and problems [J]. Popular Literature. 2015 (05)

[5] Jinhua. Huayin old cavity: the rock on the yellow soil [J]. People living. 2016 (07)

[6] Huayin City Huhu Opera Troupe to celebrate the 45th anniversary of the founding [J]. Contemporary drama. 1999 (01)

[7] Xiao Yang, Tao Long. Huayin old cavity earthquake mountain [J]. Autumn. 2011 (05) 